\title{
HEINS PROBLEM ON HARMONIC DIMENSIONS
}

\author{
Mitsuru Nakai and Toshimasa Tada \\ To the memory of Professor Nobuyuki Suita
}

\begin{abstract}
The main assertion of this paper is that for an arbitrarily given parabolic open Riemann surface $R$ there always exists a Heins surface $W_{R}$, i.e. a parabolic open Riemann surface with the single ideal boundary component, such that the harmonic dimension of $W_{R}$, i.e. the cardinal number of the set of minimal Martin boundary points of $W_{R}$, is identical with that of $R$. The result is then applied to give a simple and unified proof for the best theorem at present as an answer to the Heins problem to determine the set $\nabla$ of harmonic dimensions of all Heins surfaces obtained by collecting contributions of many authors that $\nabla$ contains the set $\mathbf{N}$ of all positive integers, the cardinal number $\aleph_{0}$ of countably infinite set, and the cardinal number $\aleph$ of continuum, i.e. $\nabla \supset \mathbf{N} \cup\left\{\aleph_{0}, \aleph\right\}$, so that $\nabla=[1, \aleph]$, the interval of cardinal numbers $\xi$ with $1 \leq \xi \leq \aleph$, when the continuum hypothesis is postulated.
\end{abstract}

\section{Introduction}

We denote by $\Delta(R)$ the Martin boundary of an open Riemann surface $R$ and by $\Delta_{1}(R)$ the set of minimal points in $\Delta(R)$. The cardinal number of $\Delta_{1}(R)$, card $\Delta_{1}(R)$ in notation, is referred to as the harmonic dimension of $R, \operatorname{dim} R$ in notation:

$$
\operatorname{dim} R=\operatorname{card} \Delta_{1}(R) \text {. }
$$

Here a few words are in order on the definition of the Martin compactification. Open Riemann surfaces are classified into two categories: hyperbolic and parabolic surfaces according to the existence or nonexistence of harmonic Green functions on them (cf. $\S 3$ below). Originally the Martin compactifications are defined by using Green functions so that these notions are basically considered for hyperbolic $R$. Let $V$ be any parametric disc $|z|<1$ in $R$ with $\bar{V}$, the closure of $V$, is given by $|z| \leq 1$. It can be seen that the defining sequence $\left(z_{n}\right)_{n \geq 1}$ in $R \backslash \bar{V}$ of a point $\zeta$ in $\Delta(R \backslash \bar{V})$ either converges to the ideal boundary of $R$ or accumulates to the relative boundary $\partial V$ of $V$. The point $\zeta$ in $\Delta(R \backslash \bar{V})$

2000 Mathematics Subject Classifications. Primary 30C25, 30C85; Secondary 31A15.

Key words and phrases. harmonic dimension, ideal boundary component, Kerékjártó-Stoïlow compactification, Martin compactification, parabolic.

Received March 9, 2004; revised August 2, 2004. 
is said to lie over the ideal boundary of $R$ or over $\partial V$ according to the former or the latter situation described above occurs. Moreover the set of points in $\Delta(R \backslash \bar{V})$ lying over $\partial V$ is identical with $\partial V$ and

$$
\Delta(R)=\Delta(R \backslash \bar{V}) \backslash \partial V
$$

is valid for any hyperbolic surface $R$ and any parametric disc $V$. Even if $R$ is parabolic, $R \backslash \bar{V}$ is hyperbolic for any parametric disc $V$ and hence $\Delta(R \backslash \bar{V})$ can be defined. It is also easy to see that

$$
\Delta\left(R \backslash \bar{V}_{1}\right) \backslash \partial V_{1}=\Delta\left(R \backslash \bar{V}_{2}\right) \backslash \partial V_{2}
$$

for any pair $\left(V_{1}, V_{2}\right)$ of parametric discs $V_{j}$ in $R(j=1,2)$. Reversing the process we define the Martin boundary $\Delta(R)$ of a parabolic surface $R$ by (2) which is independent of the choice of the parametric disc $V$ as we mentioned above. Thus (2) is valid for every open Riemann surface $R$ as a theorem for hyperbolic $R$ and as a definition for parabolic $R$. Naturally we have

$$
\Delta_{1}(R)=\Delta_{1}(R \backslash \bar{V}) \backslash \partial V
$$

for every open Riemann surface $R$. It is not important in this context but we have the inclusion $\partial V \subset \Delta_{1}(R \backslash \bar{V})$, which is sometimes convenient. In (2) and (3) we may replace $V$ by any compact subset $K$ of $R$ with positive capacity such that $R \backslash K$ is connected, where $\partial K$ here is understood as being Carathéodory relative boundary. The remark mentioned above is important since we mainly consider the harmonic dimension $\operatorname{dim} R=\operatorname{card} \Delta_{1}(R)$ for parabolic open Riemann surface $R$.

The notion of the harmonic dimension was originally introduced by Heins [4] in 1952 in connection with his study on the value distribution theory for meromorphic functions at the parabolic isolated ideal boundary component of surfaces with infinite genus and the term harmonic dimension itself was also coined by himself. It is clear that

$$
1 \leq \operatorname{dim} R \leq \aleph,
$$

where $\aleph$ is the cardinal number of continuum. We denote by $[1, \aleph]$ the set of cardinal numbers $\xi$ such that $1 \leq \xi \leq \aleph$. The interval $[1, \aleph]$ is a difficult set in general but simply $[1, \aleph]=\mathbf{N} \cup\left\{\aleph_{0}, \aleph\right\}$ if the continuum hypothesis is postulated, where $\mathbf{N}$ is the set of positive integers and $\aleph_{0}$ is the cardinal number of countably infinite set so that e.g. $\aleph_{0}=$ card $\mathbf{N}$. In any case (4) can be denoted by $\operatorname{dim} R \in$ $[1, \aleph]$.

An open Riemann surface $R$ is said to be a Heins surface if $R \in O_{G}$, the class of all parabolic open Riemann surfaces, (cf. e.g. [11], see also $\$ 3$ below) and $R$ has a single ideal boundary component, i.e. there exists only one KerékjártóStoillow ideal boundary component of $R$ (cf. e.g. [9]) so that $R$ is homeomorphic to its Richards model $R^{*} \backslash\{0\}$ ([8]), where $R^{*}$ is the compact space obtained from $\hat{\mathbf{C}} \backslash \bigcup_{n}\left(K_{n} \cup K_{n}^{\vee}\right)$ by identifying symmetric points in $\partial K_{n}$ and $\partial K_{n}^{\vee}$ with respect to the real axis. Here $\hat{\mathbf{C}}$ is the Riemann sphere and $\left(K_{n}\right)_{1 \leq n<N+1}(N<\infty$ or $N=\infty)$ is a finite or infinite sequence of mutually disjoint closed discs $K_{n}$ in the 
upper half plane of the complex plane $\mathbf{C}$ accumulating only to the origin 0 and $K_{n}^{\vee}$ is the symmetric image of $K_{n}$ with respect to the real axis. The space $R^{*}$ is the Kerékjártó-Stoïlow compactification of $R$. The condition that $R$ has a single ideal boundary component is also characterized by the fact that $R \backslash X$ has only one relatively noncompact connected component for any compact subset $X$ of $R$. We denote by $\mathscr{H}$ the class of Heins surfaces so that $\mathscr{H} \subset O_{G}$.

The punctured sphere $\hat{\mathbf{C}} \backslash\{0\}$ is the simplest and a typical Heins surface and the fact that

$$
\operatorname{dim}(\hat{\mathbf{C}} \backslash\{0\})=1
$$

is referred to mainly by French mathematicians such as Bouligand and Brelot as the Picard principle but chronologically the fact itself was found by Bôcher (cf. e.g. [1]) much earlier than Picard. Nevertheless we rather prefer to continue to call (5) as the Picard principle for several reasons. Looking at Richards model $R^{*}$ of $R \in \mathscr{H}$, one might feel that the Picard principle remains to be true for every $R \in \mathscr{H}$ but Heins pointed out that this is only true for $R \in \mathscr{H}$ of finite genus and is no longer the case in general, i.e. there is an $R \in \mathscr{H}$ of infinite genus with $\operatorname{dim} R>1$, and thus proposed the following problem in the same paper [4] cited above. Let

$$
\nabla:=\{\operatorname{dim} R: R \in \mathscr{H}\},
$$

the set of harmonic dimensions $\operatorname{dim} R$ for all $R \in \mathscr{H}$.

The Heins Problem. Determine the set $\nabla$ of harmonic dimensions.

As mentioned above Heins [4] (1952) himself showed that $\mathbf{N} \subset \nabla$. Then Kuramochi [5] (1954) claimed that $\aleph_{0} \in \nabla$ (cf. also Cornea [3] (1958), Segawa [12] (1981), Nakai-Sario [7] (1985)). Finally in this direction $\aleph \in \nabla$ was shown by Constantinescu-Cornea [2] (1959). Thus the present best knowledge on the above Heins problem is summarized as follows:

THEOREM A. The range $\nabla$ of the harmonic dimension $\operatorname{dim}$ as a mapping of the family $\mathscr{H}$ of Heins surfaces to cardinal numbers satisfies the following inclusion relations:

$$
\mathbf{N} \cup\left\{\aleph_{0}, \aleph\right\} \subset \nabla \subset[1, \aleph]
$$

If the continuum hypothesis: $\mathbf{N} \cup\left\{\aleph_{0}, \aleph\right\}=[1, \aleph]$ is postulated, then (6) is reduced to the identity $\nabla=[1, \aleph]$ and the Heins problem is completely over by this identity. Therefore the problem left is to settle whether there is an $R \in \mathscr{H}$ with $\operatorname{dim} R=\xi$ or not for an arbitrarily given cardinal number $\xi$ with $\aleph_{0}<$ $\xi<\aleph$. In the present paper, however, we are only concerned with the proof of the above theorem A. Of course putting 6 proofs cited above together produces a proof for (6) but any one of these 6 proofs is treating only an relevant individual case of $\mathbf{N} \subset \nabla, \aleph_{0} \in \nabla$, or $\aleph \in \nabla$ separately and cannot be applied to other cases. Thus it is desirable to prove that $\xi \in \nabla$ if $\xi \in \mathbf{N} \cup\left\{\aleph_{0}, \aleph\right\}$ in a unified 
fashion independent of the choice of $\xi \in \mathbf{N}, \xi=\aleph_{0}$, or $\xi=\aleph$. The secondary purpose of this paper is to give such a proof in $\$ 5$ as an application of a general result mentioned below as our main theorem proving which is our primary purpose of the present paper.

\section{Construction of canonical Heins surfaces}

Given an arbitrary $R \in O_{G}$ and we will construct in a fixed procedure a Riemann surface $W_{R} \in \mathscr{H}$ with $\operatorname{dim} W_{R}=\operatorname{dim} R$ which will be called a canonical Heins surface associated with $R$. As the first step of our fixed procedure of constructing $W_{R}$ we start with fixing a regular exhaustion $\left(R_{n}\right)_{n \geq 0}$ of $R$ with $R_{0}$ a parametric disc such that $\bar{R}_{0}=\{|z| \leq 1\}$, i.e. a sequence $\left(R_{n}\right)_{n \geq 0}$ of relatively compact subregion $R_{n}$ bounded by a finite number of disjoint analytic Jordan curves such that $R \backslash \bar{R}_{n}$ has no relatively compact component for each $n \geq 0$ and moreover $\bar{R}_{n} \subset R_{n+1}(n \geq 0)$ and $R=\bigcup_{n \geq 0} R_{n}$. Let

$$
R_{n+1} \backslash \bar{R}_{n}=\bigcup_{1 \leq j \leq N_{n}} R_{n j} \quad(n \geq 1)
$$

be the decomposition of $R_{n+1} \backslash \bar{R}_{n}$ into connected components $R_{n j}\left(1 \leq j \leq N_{n}\right)$. Choose and then fix an arbitrary parametric disc $B_{n j}=\{|z|<1\}$ with $\bar{B}_{n j} \subset R_{n j}$ and then the concentric disc $D_{n j}=\{|z|<1 / 2\}$ to $B_{n j}$ and finally the radial slit $\gamma_{n j}=\left\{z \in B_{n j}: 0 \leq \operatorname{Re} z \leq r_{n}, \operatorname{Im} z=0\right\} \quad\left(0<r_{n}<1 / 4\right)$ in $D_{n j} \subset B_{n j}$ which will be able to be made short by taking $r_{n}$ enough small (cf. (7) below). For simplicity, set

$$
B_{n}:=\bigcup_{1 \leq j \leq N_{n}} B_{n j}, \quad D_{n}:=\bigcup_{1 \leq j \leq N_{n}} D_{n j}, \quad \gamma_{n}:=\bigcup_{1 \leq j \leq N_{n}} \gamma_{n j} \quad(n \geq 1) .
$$

Moreover we put

$$
B:=\bigcup_{n \geq 1} B_{n}, \quad D:=\bigcup_{n \geq 1} D_{n}, \quad \gamma:=\bigcup_{n \geq 1} \gamma_{n}, \quad \Omega:=R \backslash \bar{R}_{0} .
$$

Let $B_{n j}^{\prime}:=\{|z-3 j|<1\} \quad$ be in $\mathbf{C}$ and $\gamma_{n j}^{\prime}:=\left\{z \in B_{n j}^{\prime}: 0 \leq \operatorname{Re}(z-3 j) \leq r_{n}\right.$, $\operatorname{Im} z=0\} \quad\left(1 \leq j \leq N_{n}, n \geq 1\right)$ and

$$
B_{n}^{\prime}:=\bigcup_{1 \leq j \leq N_{n}} B_{n j}^{\prime}, \quad \gamma_{n}^{\prime}:=\bigcup_{1 \leq j \leq N_{n}} \gamma_{n j}^{\prime} \quad(n \geq 1) .
$$

As above we also set

$$
B^{\prime}:=\bigcup_{n \geq 1} B_{n}^{\prime}, \quad \gamma^{\prime}:=\bigcup_{n \geq 1} \gamma_{n}^{\prime} .
$$

The region $S_{n}:=\hat{\mathbf{C}} \backslash \gamma_{n}^{\prime}$ is referred to as a finite chain of handles. Two of these regions are viewed to be disjoint if they are different: $S_{n} \cap S_{m}=\emptyset(n \neq m)$. We set $S:=\bigcup_{n \geq 1} S_{n}$, a set of handles. A finite chain $S_{n}$ of handles is viewed as being getting smaller and smaller by making $r_{n} \downarrow 0$ for each $n \geq 1$.

Fix a point $a$ in $\Omega \backslash \bar{B}$ and let $k_{n}:=k\left(\{a\} \cup \partial B_{n} ; \Omega \backslash \bar{D}\right) \geq 1$ be the Harnack constant of the compact set $\{a\} \cup \partial B_{n}$ with respect to the region $\Omega \backslash \bar{D}(n \geq 1)$ : 
$h\left(z^{\prime}\right) \leq k_{n} h\left(z^{\prime \prime}\right)$ for every pair $\left(z^{\prime}, z^{\prime \prime}\right)$ of points $z^{\prime}$ and $z^{\prime \prime}$ in $\{a\} \cup \partial B_{n}$ and $h$ in $H P(\Omega \backslash \bar{D})$, the class of nonnegative harmonic functions $h$ on $\Omega \backslash \bar{D}$. Let $\omega=$ $\omega\left(\cdot, \gamma_{n}, \Omega \backslash \gamma_{n}\right)$ be the harmonic measure of $\gamma_{n}$ with respect to the region $\Omega \backslash \gamma_{n}$ : $\omega=\inf s$ on $\Omega \backslash \gamma_{n}$, where the infimum is taken with respect to nonnegative superharmonic functions $s$ on $\Omega$ such that $s \geq 1$ on $\gamma_{n}$. It is clear that

$$
\lim _{r_{n} \downarrow 0} \omega\left(\cdot, \gamma_{n}, \Omega \backslash \gamma_{n}\right)=0
$$

on $\Omega \backslash \gamma_{n}$. Therefore it is possible to take an $r_{n} \in(0,1 / 4)$ so small that

$$
\omega\left(a, \gamma_{n}, \Omega \backslash \gamma_{n}\right)<\frac{1}{2^{n+1} k_{n}^{2}} \quad(n \geq 1) .
$$

We hence hereafter assume that the union $\gamma$ of the unions $\gamma_{n}(n \geq 1)$ of slits $\gamma_{n j}\left(1 \leq j \leq N_{n}\right)$ has been chosen and fixed so small as to satisfy the condition (7) above.

Now we attach the set $S$ of chains $S_{n}$ of handles $(n \geq 1)$ to $R$ as follows. We view each $B_{n j}$ in $B_{n}$ in $B$ and each $B_{n j}^{\prime}$ in $B_{n}^{\prime}$ in $B^{\prime}$ are copies of the same unit disc and identify each $\gamma_{n j}$ in $\gamma_{n}$ in $\gamma$ with $\gamma_{n j}^{\prime}$ in $\gamma_{n}^{\prime}$ in $\gamma^{\prime}\left(1 \leq j \leq N_{n}\right.$, $n \geq 1$ ). We take $W:=R \backslash \gamma$ and connect $S$ to $W$ by joining $S_{n}$ to $W$ crosswise along each edge of each $\gamma_{n j}=\gamma_{n j}^{\prime}\left(1 \leq j \leq N_{n}\right)$ for every $n \geq 1$, i.e. connect $S_{n}$ to $W$ by identifying the upper (lower, resp.) edge $\gamma_{n j}^{+}\left(\gamma_{n j}^{-}\right.$, resp.) of $\gamma_{n j}$ with the lower (upper, resp.) edge $\left(\gamma_{n j}^{\prime}\right)^{-}\left(\left(\gamma_{n j}^{\prime}\right)^{+}\right.$, resp.) of $\gamma_{n j}^{\prime}$ for each $1 \leq j \leq N_{n}$ and for each $n \geq 1$. The resulting surface will be denoted by $W_{R}$. In $W_{R}$ each $S_{n}$ is viewed as a relatively compact regular subregion of $W_{R}$ whose relative boundary $\partial S_{n}$ in $W_{R}$ is the union $\gamma_{n}^{ \pm}$of a finite number of mutually disjoint analytic Jordan curves $\gamma_{n j}^{+} \cup \gamma_{n j}^{-}\left(1 \leq j \leq N_{n}\right)$, where $\gamma_{n j}^{+}\left(\gamma_{n j}^{-}\right.$, resp. $)$is as already mentioned above the upper (lower, resp.) edge of the slit $\gamma_{n j}=\gamma_{n j}^{\prime}\left(1 \leq j \leq N_{n}\right)$. Recall that at each point $\zeta$ of $W_{R} \backslash \gamma$ the original local parameter $z$ in $R$ or $S$ is adopted as that for $W_{R}$; at each point $\zeta$ at $\gamma$ except for the end points of each $\gamma_{n j}$ we take $z-\zeta$ as the local parameter at $\zeta$ where $z$ is the natural plane coordinate in $B_{n j}$ and also in $B_{n j}^{\prime}$ containing $\zeta$; finally at each end point $\zeta$ of $\gamma_{n j}$ in $B_{n j}$ and also in $B_{n j}^{\prime}$ with their original coordinate $z$ as above we choose the function $\sqrt{z-\zeta}$ with $\sqrt{1}=1$ as the local parameter at $\zeta$. Because of such definition of the conformal structure of $W_{R}$ the set $\gamma_{n j}^{+} \cup \gamma_{n j}^{-}$becomes an analytic Jordan curve in the Carathéodory compactification of $B_{n j} \backslash \gamma_{n j}$ whose boundary is defined to be the set of boundary elements of $B_{n j} \backslash \gamma_{n j}$ embedded in $W_{R}$ in the sense of Carathéodory.

The surface $W_{R}$ has the following particular properties to prove which is the main purpose of this paper as announced in the introduction. After a preparation in $\S 3$ the proof will be given in $\S 4$ below.

The MaIn TheOREM. The surface $W_{R}$ associated with an arbitrarily given surface $R$ in the parabolic class $O_{G}$ is a Heins surface whose harmonic dimension is identical with that of $R$ :

$$
\operatorname{dim} W_{R}=\operatorname{dim} R .
$$


The Riemann surface $W_{R}$ constructed from a given Riemann surface $R$ in $O_{G}$ in the fixed fashion described above will be referred to as a canonical Heins surface associated with $R$. We use the characterization for $W_{R}$ to have a single ideal boundary component that there exists an exhaustion $\left(X_{n}\right)_{n>0}$ of $W_{R}$ such that $W_{R} \backslash \bar{X}_{n}$ is connected for every $n \geq 0$. The modification $W_{R}$ of $R$ is so performed as to ensure the possibility of constructing such an exhaustion $\left(X_{n}\right)_{n>0}$ described above. One of the roles of the requirement (7) is to guarantee for $W_{R}$ to inherit the parabolicity from the fact that $R$ is parabolic. Thus the part $W_{R} \in O_{G}$ of the proof of $W_{R} \in \mathscr{H}$, the class of Heins surfaces, is almost straight forward. Really important role of the condition (7) is to assure the validity of the identity (8), to prove which is the main part of the proof of the above theorem given in $\$ 4$ below.

Recall that the Heins problem is to determine the range set $\nabla=\{\operatorname{dim} R$ : $R \in \mathscr{H}\}$ of the mapping $\operatorname{dim}: \mathscr{H} \rightarrow[1, \aleph]$. Since $\mathscr{H} \subset O_{G}, \quad \nabla \subset\{\operatorname{dim} R:$ $\left.R \in O_{G}\right\}$. The identity (8) implies $\left\{\operatorname{dim} R: R \in O_{G}\right\}=\left\{\operatorname{dim} W_{R}: R \in O_{G}\right\} \subset \nabla$. Thus we can conclude that

$$
\nabla=\left\{\operatorname{dim} R: R \in O_{G}\right\} .
$$

Hence the Heins problem can be rephrased as follows: determine the range of the mapping $\operatorname{dim}: O_{G} \rightarrow[1, \aleph]$. In this formulation a unified simple proof of Theorem A can be instantaneously given as will be observed later in $§ 5$.

\section{Parabolic surfaces and a maximum principle}

In the classification theory of Riemann surfaces (cf. e.g. [11]), the class of harmonic (nonnegative harmonic, bounded harmonic, resp.) functions on an open Riemann surface $F$ is denoted by $H(F)\left(H P(F), H B(F)\right.$, resp.). The class $O_{G}$ is the totality of every parabolic surface $F$, i.e. an open Riemann surface $F$ on which there is no Green function (cf. e.g. [11]). Let $F_{0}:=\{|z|<1\}$ be a parametric disc on $F$ such that $\bar{F}_{0}=\{|z| \leq 1\} \subset F$. An open Riemann surface $F$ is parabolic, i.e. $F \in O_{G}$ in notation, if and only if there exists an Evans function $h$ on $F \backslash \bar{F}_{0}$ (cf. [6], see also [10], [11]) characterized by the conditions that $h \in H P\left(F \backslash \bar{F}_{0}\right) \cap C\left(F \backslash F_{0}\right)$ with $h \mid \partial F_{0}=0$ and $\lim _{z \rightarrow \infty_{F}} h(z)=+\infty$, where $\infty_{F}$ is the Alexandroff ideal boundary point of $F$. The harmonic measure $\omega=\omega\left(\cdot, \beta, F \backslash \bar{F}_{0}\right)$ of the ideal boundary $\beta$ of $F$ relative to $F \backslash \bar{F}_{0}$ is given by

$$
\omega=\inf _{s \in \mathscr{S}} s
$$

on $F \backslash \bar{F}_{0}$, where $\mathscr{S}$ is the class of nonnegative superharmonic functions $s$ on $F \backslash \bar{F}_{0}$ such that $s \geq 1$ on $F$ outside some compact subset of $F$ containing $\bar{F}_{0}$. The above definition of $\omega$ assures that $\omega \in H P\left(F \backslash \bar{F}_{0}\right)$. As for the behavior of $\omega$ at the relative boundary $\partial F_{0}$ of $F \backslash \bar{F}_{0}$, we see that $\omega$ vanishes continuously on $\partial F_{0}$. In fact, let $F_{1}$ be an arbitrary regular subregion of $F$ containing $\bar{F}_{0}$ and an $s_{1} \in \mathscr{S}$ be such that $s_{1} \in H\left(F_{1} \backslash \bar{F}_{0}\right) \cap C\left(F \backslash F_{0}\right), \quad s_{1} \mid F \backslash F_{1}=1$, and $s_{1} \mid \partial F_{0}=0$. Such an $s_{1}$ can be found by solving the Dirichlet problem on $F_{1} \backslash \bar{F}_{0}$ with 
boundary values 1 on $\partial F_{1}$ and 0 on $\partial F_{0}$. Then $0 \leq \omega \leq s_{1}$ on $F \backslash \bar{F}_{0}$, which implies that

$$
0 \leq \limsup _{z \in F \backslash \bar{F}_{0}, z \rightarrow \zeta} \omega(z) \leq \lim _{z \in F \backslash \bar{F}_{0}, z \rightarrow \zeta} s_{1}(z)=0
$$

for every $\zeta \in \partial F_{0}$, i.e. $\omega$ has continuously vanishing boundary values on $\partial F_{0}$. It is known that $F \in O_{G}$ is also characterized by that $\omega=0$ on $F \backslash \bar{F}_{0}$ (cf. e.g. [11]).

In general, no matter whether $F \in O_{G}$ or not, we have the identity

$$
\left(\sup _{F \backslash \bar{F}_{0}} \omega\right) \omega=\omega
$$

on $F \backslash \bar{F}_{0} . \quad$ In fact, let $\lambda:=\sup _{F \backslash \bar{F}_{0}} \omega$. Observe that $\min (s, 1) \in \mathscr{S}$ along with $s \in \mathscr{S}$ so that $0 \leq \omega \leq 1$ and hence $0 \leq \lambda \leq 1$. Take an arbitrary real number $\varepsilon>0$. Since the harmonic function $\omega /(\lambda+\varepsilon)<1$ on $F \backslash \bar{F}_{0}$ and $\omega /(\lambda+\varepsilon)$ has vanishing boundary values on $\partial F_{0}$ as we saw above, the minimum principle for superharmonic functions implies that $s \geq \omega /(\lambda+\varepsilon)$ on $F \backslash \bar{F}_{0}$ for any $s \in \mathscr{S}$ so that $\omega \geq \omega /(\lambda+\varepsilon)$ or $(\lambda+\varepsilon) \omega \geq \omega$ on $F \backslash \bar{F}_{0}$. On letting $\varepsilon \downarrow 0$ we have $\lambda \omega \geq \omega$ on $F \backslash \bar{F}_{0}$. This with the trivial fact that $\lambda \omega \leq \omega$ on $F \backslash \bar{F}_{0}$ yields (10) above. Hence in particular $F \notin O_{G}$ is equivalent to

$$
\sup _{F \backslash \bar{F}_{0}} \omega=1 .
$$

Let $G$ be a subregion of an $F \in O_{G}$ such that $\bar{G} \subset F \backslash \bar{F}_{0}$ and $s$ be a superharmonic (subharmonic, resp.) function on $G$ bounded from below (above, resp.) on $G$. Then the following minimum (maximum, resp.) principle is valid: if

$$
\liminf _{z \in G, z \rightarrow \zeta} s(z) \geq 0 \quad\left(\limsup _{z \in G, z \rightarrow \zeta} s(z) \leq 0, \text { resp. }\right)
$$

for any $\zeta \in \partial G$, then $s \geq 0(s \leq 0$, resp.) on $G$. These are also referred to as the comparison principle. To prove these we can assume $\bar{G}$ is not compact in $F \backslash \bar{F}_{0}$. Otherwise these are reduced to the mere usual comparison principle. We take an Evans function $h$ on $F \backslash \bar{F}_{0}$ and an arbitrary positive number $\varepsilon$ and then consider the function $s+\varepsilon h(s-\varepsilon h$, resp.) on $G$. Then we see that

$$
\liminf _{z \in G, z \rightarrow \zeta}(s(z)+\varepsilon h(z)) \geq 0 \quad\left(\limsup _{z \in G, z \rightarrow \zeta}(s(z)-\varepsilon h(z)) \leq 0, \text { resp. }\right)
$$

for every $\zeta \in \partial G$ by the assumption on $s$ and also for $\zeta=\infty_{G}$, the Alexandroff point of $G$ defined to be the single point left in $\operatorname{cl}[G] \backslash(G \cup \partial G)$ with $\operatorname{cl}[G]$ the closure of $G$ in the Alexandroff compactification $F \cup\left\{\infty_{F}\right\}$ of $F$, as consequences of $s$ being bounded below (above, resp.) and the behavior of $h$ at $\infty_{F}$ and hence at $\infty_{G}$ : $\lim _{z \in G, z \rightarrow \infty_{G}} h(z)=+\infty$. From the above boundary behavior of $s+\varepsilon h$ $(s-\varepsilon h$, resp.) it follows that $s+\varepsilon h \geq 0(s-\varepsilon h \leq 0$, resp.) on $G$ as a consequence of the usual minimum (maximum, resp.) principle. On letting $\varepsilon \downarrow 0$, we deduce the desired conclusion. The result applied to $u \in H B(G)$ will be referred to as 
the maximum principle: if the upper boundary values of $u \leq c$ on $\partial G$, then $u \leq c$ on $G$.

\section{Proof of the main theorem}

We now prove the main theorem: $W_{R} \in \mathscr{H}$ and $\operatorname{dim} W_{R}=\operatorname{dim} R . \quad$ We will use the notations introduced in $\$ 2$ without further reviewing them. We denote by $X_{n}$ the relatively compact subregion of $W_{R}$ bounded by $\partial R_{n}(n \geq 1)$ and also $X_{0}:=R_{0}$ for $n=0$ so that

$$
R_{n}=\left(X_{n} \backslash \bigcup_{1 \leq v \leq n-1} S_{v}\right) \cup \bigcup_{1 \leq v \leq n-1} \gamma_{v} \quad(n \geq 0),
$$

where $\bigcup_{1 \leq v \leq n-1} S_{v}$ and $\bigcup_{1 \leq v \leq n-1} \gamma_{v}$ are understood to be the empty set for $n=0$ and 1 . Then the sequence $\left(X_{n}\right)_{n \geq 0}$ is a regular exhaustion of $W_{R}$. Since $X_{0}=R_{0}$ is a disc, $W_{R} \backslash \bar{X}_{0}$ is connected. Moreover, although $R \backslash \bar{R}_{n}$ may consist of at least one and at most $N_{n}$ components and may not be connected, since these are joined by the chain $S_{n}$ of handles in $W_{R} \backslash \bar{X}_{n}$, we see that $W_{R} \backslash \bar{X}_{n}$ is connected not only for $n=0$ but also for every $n \geq 0$. By the existence of an exhaustion $\left(X_{n}\right)_{n \geq 0}$ of $W_{R}$ with the property that $W_{R} \backslash \bar{X}_{n}$ is connected for every $n \geq 0$, we conclude that $W_{R}$ has only one ideal boundary component. Therefore we only have to show that $W_{R} \in O_{G}$ in order to conclude that $W_{R}$ is a Heins surface, i.e. $W_{R} \in \mathscr{H}$. For the purpose we take the harmonic measure $\omega=\omega(\cdot, \beta, X)$ of the ideal boundary $\beta$ of $W_{R}$ with respect to the region $X:=W_{R} \backslash \bar{X}_{0}$ and we have to show that $\omega=0$ on $X$. Contrary to the assertion, assume that $\omega>0$ on $X$ so that by (11)

$$
\sup _{X} \omega=\sup _{z \in X} \omega(z, \beta, X)=1 .
$$

Let $Y_{n}$ be the relatively compact subregion of $W_{R}$ bounded by $\partial B_{n}(n \geq 1)$ so that $Y_{n} \supset \bar{S}_{n}$. Recall that $W=R \backslash \gamma$ and $\Omega=R \backslash \bar{R}_{0}$. Observe that $\partial W=\gamma^{ \pm}:=$ $\bigcup_{n \geq 1} \gamma_{n}^{ \pm}$considered in $W_{R}$ but $\partial W=\gamma$ considered in $R$. Since

$$
\omega \leq \sum_{n \geq 1} \omega\left(\cdot, \gamma_{n}, \Omega \backslash \gamma\right) \leq \sum_{n \geq 1} \omega\left(\cdot, \gamma_{n}, \Omega \backslash \gamma_{n}\right) \leq \sum_{n \geq 1} \frac{1}{2^{n+1} k_{n}} \leq \frac{1}{2}
$$

on $\partial B$ and trivially on $\partial X_{0}$, the maximum principle yields that $\omega \leq 1 / 2$ on $\Omega \backslash B$. In particular, $\omega \leq 1 / 2$ on $\partial B$ implies that $\omega \leq 1 / 2$ on $\bigcup_{n \geq 1} Y_{n}$ so that $\omega \leq 1 / 2$ on $X=W_{R} \backslash \bar{X}_{0}$, contradicting (12) so that $W_{R} \in O_{G}$.

Having finished the proof of $W_{R} \in \mathscr{H}$ we turn to the proof of the essential part $\operatorname{dim} W_{R}=\operatorname{dim} R$. In general, let $G$ be a subregion of a Riemann surface and $\Gamma \subset \partial G$. We use the notation $H P(G ; \Gamma)$ for the class of functions $u \geq 0$ continuous on $G \cup \Gamma$ and harmonic on $G$ with vanishing boundary values on $\Gamma$ :

$$
H P(G ; \Gamma)=\{u \in H P(G) \cap C(G \cup \Gamma): u \mid \Gamma=0\} .
$$

Using this notation we first show that 


$$
H P(\Omega ; \partial \Omega) \simeq H P(\Omega \backslash \gamma ; \partial \Omega \cup \gamma),
$$

which means that there exists a positively homogeneous additive bijective mapping of the half module $H P(\Omega ; \partial \Omega)$ onto the half module $H P(\Omega \backslash \gamma ; \partial \Omega \cup \gamma)$. Let $u \in H P(\Omega ; \partial \Omega)$ and $H_{n} u$ be the bounded harmonic function on $\Omega \backslash \gamma$ such that $H_{n} u$ has vanishing boundary values on $\partial \Omega \cup\left(\gamma \backslash \gamma_{n}\right)$ and the boundary values $u$ on $\gamma_{n}(n \geq 1)$. The possibility of constructing such an $H_{n} u$ and its uniqueness is seen as follows. By solving the Dirichlet problem on $\Omega_{m} \backslash \bigcup_{1 \leq v \leq m-1} \gamma_{v}(m \geq$ $n+2)$ with $\Omega_{m}:=R_{m} \backslash \bar{R}_{0}$, we can take an $h_{n m}=h_{m} \in H\left(\Omega_{m} \backslash \bigcup_{1 \leq v \leq m-1} \gamma_{v}\right) \cap$ $C\left(\bar{\Omega}_{m}\right)$ such that $h_{m} \mid \partial \Omega_{m} \cup\left(\bigcup_{1 \leq v \leq m-1, v \neq n} \gamma_{v}\right)=0$ and $h_{m} \mid \gamma_{n}=u$. The maximum principle assures that the sequence $\left(h_{m}\right)_{m \geq n+2}$ is increasing and uniformly bounded on every compact subset of $\bar{\Omega}$ and a fortiori

$$
h:=\lim _{m \uparrow \infty} h_{m} \in H B(\Omega \backslash \gamma) \cap C(\bar{\Omega})
$$

exists with $h \mid \partial \Omega \cup\left(\gamma \backslash \gamma_{n}\right)=0$ and $h \mid \gamma_{n}=u$. In view of $R \in O_{G}$, the maximum principle (cf. §3) yields that such an $h$ is uniquely determined and therefore $h=H_{n} u$. Without loss of generality we assume that $u(a)=1$. Then the Harnack inequality implies that $u \leq k_{n}$ on $\partial B_{n}$ and hence on $\gamma_{n}$. Then by (7), $H_{n} u(a)<1 / 2^{n+1} k_{n}$ and again by the Harnack inequality $H_{n} u<1 / 2^{n+1}$ on $\partial B_{n}$. Hence $H_{n} u<1 / 2^{n+1}$ on $\Omega \backslash \bar{B}_{n}$ and

$$
H u:=\sum_{n \geq 1} H_{n} u<\frac{1}{2}
$$

on $\Omega \backslash \bar{B}$. The operator $H$ defined above is thus seen to be a positively homogeneous additive mapping of the halfmodule $H P(\Omega ; \partial \Omega)$ to that $H P(\Omega \backslash \gamma ; \partial \Omega)$ and $H u(u \in H P(\Omega ; \partial \Omega))$ is characterized as the unique function in $H P(\Omega \backslash \gamma ; \partial \Omega)$ $\cap H B(\Omega \backslash \bar{B}) \cap C(\bar{\Omega})$ such that $H u \mid \gamma=u$. Let

$$
H P(\Omega ; \partial \Omega) \ominus H P(\Omega ; \partial \Omega)=\{u-v: u, v \in H P(\Omega ; \partial \Omega)\}
$$

the linearlized space generated by $H P(\Omega ; \partial \Omega)$, and $H P(\Omega \backslash \gamma ; \partial \Omega) \ominus H P(\Omega \backslash \gamma ; \partial \Omega)$ be similarly defined as above. We insert here a remark that we can further extend the domain of definition of $H$ so as to be a linear operator from the linear space $H P(\Omega ; \partial \Omega) \ominus H P(\Omega ; \partial \Omega)$ to that $H P(\Omega \backslash \gamma ; \partial \Omega) \ominus H P(\Omega \backslash \gamma ; \partial \Omega)$ by the relation

$$
H(u-v):=H u-H v \quad(u, v \in H P(\Omega ; \partial \Omega)) .
$$

It is aesy to see that $H(u-v)$ depends solely upon the function $u-v$ and does not depend on the particular decomposition $u-v$, i.e. if $u, v, u^{\prime}, v^{\prime}$ are in $H P(\Omega ; \partial \Omega)$ and $u-v=u^{\prime}-v^{\prime}$, then $H(u-v)=H\left(u^{\prime}-v^{\prime}\right)$. As before $H h(h \in$ $H P(\Omega ; \partial \Omega) \ominus H P(\Omega ; \partial \Omega))$ is seen to be characterized as the unique function in $(H P(\Omega \backslash \gamma ; \partial \Omega) \ominus H P(\Omega \backslash \gamma ; \partial \Omega)) \cap H B(\Omega \backslash \bar{B}) \cap C(\bar{\Omega})$ such that $H h \mid \gamma=h$. Using $H$ we now consider the operator $T$ given by

$$
T u:=u-H u
$$


on $\Omega \backslash \gamma . \quad$ It is seen that $T u \in H P(\Omega \backslash \gamma ; \partial \Omega \cup \gamma)$ and $T$ is a positively homogeneous (i.e. $T(c u)=c T u$ for nonnegative real numbers $c$ ) additive (i.e. $T\left(u_{1}+u_{2}\right)=$ $\left.T u_{1}+T u_{2}\right)$ mapping of $H P(\Omega ; \partial \Omega)$ to $H P(\Omega \backslash \gamma ; \partial \Omega \cup \gamma)$.

We first show that $T: H P(\Omega ; \partial \Omega) \rightarrow H P(\Omega \backslash \gamma ; \partial \Omega \cup \gamma)$ is injective. Take two functions $u$ and $v$ arbitrarily in $H P(\Omega ; \partial \Omega)$ and assume that $T u=T v$, i.e. $u-H u=v-H v$ or $u-v=H(u-v)$. We have to show that $u=v$ on $\Omega$. Since $H(u-v)=u-v$ is harmonic on each $B_{n}$ and

$$
|H(u-v)| \leq H u+H v \leq \frac{u(a)}{2}+\frac{v(a)}{2}
$$

on $\Omega \backslash \bar{B}$, we see that $|H(u-v)| \leq u(a) / 2+v(a) / 2$ on $\Omega$. Since $H(u-v)$ has vanishing boundary values on $\partial \Omega$, the maximum principle implies that $H(u-v)=0$ on $\Omega$ so that $u-v=H(u-v)=0$ on $\Omega$ and thus $u=v$ on $\Omega$.

Next we show that $T: H P(\Omega ; \partial \Omega) \rightarrow H P(\Omega \backslash \gamma ; \partial \Omega \cup \gamma)$ is surjective. Take an arbitrary $v \in H P(\Omega \backslash \gamma ; \partial \Omega \cup \gamma)$ and we are to find a $u \in H P(\Omega ; \partial \Omega)$ such that $T u=v$. We can view that $v$ is subharmonic on $\Omega$ by setting $v=0$ on $\gamma$. Again we may suppose that $v(a)=1$. Take the function $w \in H P(\Omega \backslash \gamma) \cap C(\bar{\Omega})$ given by

$$
w:=\sum_{n \geq 1} 4 k_{n} \omega\left(\cdot, \gamma_{n}, \Omega \backslash \gamma\right)
$$

Fix an arbitrary $m \in \mathbf{N}$ and set

$$
w_{m}:=\sum_{n=1}^{m} 4 k_{n} \omega\left(\cdot, \gamma_{n}, \Omega \backslash \gamma\right) .
$$

Then $w_{m} \mid \gamma_{n} \leq 4 k_{n}$ and, by (7) and the definition of $k_{n}$, we see that

$$
w_{m} \mid \partial B_{n} \leq \sum_{v=1}^{m} 4 k_{v} \cdot \frac{k_{v}}{2^{v+1} k_{v}^{2}}<2 .
$$

Since $w_{m}$ is bounded on $\Omega \backslash \bar{B}$ we can conclude that $w_{m} \leq 2$ on $\Omega \backslash \bar{B}$. By letting $m \uparrow \infty$ we finally conclude that

$$
w \leq 2
$$

on $\Omega \backslash \bar{B}$. Hence $s:=v+w \in H P(\Omega \backslash \gamma) \cap C(\overline{\mathbf{\Omega}})$ and $s \mid \gamma_{n}=4 k_{n}$, and

$$
s\left|\partial B_{n} \leq k_{n}+2 \leq 3 k_{n}<4 k_{n}=s\right| \gamma_{n}
$$

assures that $s\left|B_{n} \leq 4 k_{n}=s\right| \gamma_{n}$, which shows that $s$ is a superharmonic majorant of $v$ on $\Omega$. Hence there is the least harmonic majorant $u$ of $v$ on $\Omega$ such that $v \leq u \leq s$ on $\Omega$. Hence in particular $u \in H P(\Omega ; \partial \Omega)$. Then, since $H u \leq H s=$ $H w=w$, we have

$$
|T u-v| \leq(u-v)+H u \leq(s-v)+H u \leq w+w=2 w
$$

on $\Omega$. Observe that $T u-v=0$ on $\gamma \cup \partial \Omega,|T u-v| \leq 2 w \leq 4$ on $\Omega \backslash \bar{B}$ so that $T u-v$ is bounded and harmonic on $\Omega \backslash \gamma$ with vanishing boundary values, i.e. 
$T u-v=0$ on $\partial \Omega \cup \gamma$. Hence $T u=v$. Thus we have established the relation (13).

In $W_{R}$ the slit $\gamma_{n j}$ was viewed as an analytic Jordan curve $\gamma_{n j}^{+} \cup \gamma_{n j}^{-}$so that $B_{n j} \backslash \gamma_{n j}$ was also viewed as an annulus $A_{n j}$ bounded by analytic Jordan curves $\partial B_{n}$ and $\gamma_{n j}^{+} \cup \gamma_{n j}^{-}$and $S_{n}$ was viewed as a relatively compact subregion bounded by the finite union $\gamma_{n}^{ \pm}=\bigcup_{1 \leq j \leq N_{n}}\left(\gamma_{n j}^{+} \cup \gamma_{n j}^{-}\right)$of mutually disjoint analytic Jordan curves $\gamma_{n j}^{+} \cup \gamma_{n j}^{-}$. Recall that $X=W_{R} \backslash \bar{X}_{0}$. We also set $\gamma^{ \pm}:=\bigcup_{n \geq 1} \gamma_{n}^{ \pm}$. We next maintain the following relation which is a counterpart of (13). Namely,

$$
H P(X ; \partial X) \simeq H P\left(X \backslash \bar{S} ; \partial X \cup \gamma^{ \pm}\right) .
$$

The proof of the above relation is essentially the same as that for (13). For the sake of completeness we briefly state the outline of the proof of (14) mimicking the proof of (13). Using the same notation as before let $H_{n} u$, for each integer $n \geq 1$, be the bounded harmonic function on $Y:=X \backslash \bar{S}$ such that $H_{n} u$ has boundary values zero on $\partial X \cup\left(\gamma^{ \pm} \backslash \gamma_{n}^{ \pm}\right)$and $u$ on $\gamma_{n}^{ \pm}$for any arbitrarily given $u$ in $H P(X ; \partial X)$. The unique existence of such an $H_{n} u \in H B(Y)$ for each $u \in H P(X ; \partial X)$ can be seen by exactly the same fashion as was employed in the proof of (13). Without loss of generality we may assume that $u(a)=1$ in order to show the convergence of the sequence $\sum_{j \geq 1} H_{j} u$. Then the Harnack inequality shows that $u \leq k_{n}$ on $\partial B_{n}$ and hence on $\gamma_{n}^{ \pm}$. By (7), $H_{n} u(a)<1 /$ $\left(2^{n+1} k_{n}\right)$ and again by the Harnack inequality $H_{n} u<1 / 2^{n+1}$ on $\partial B_{n}$. Then $H_{n} u<1 / 2^{n+1}$ on $Y \backslash \bar{B}_{n}^{\prime \prime}$ and a fortiori

$$
H u:=\sum_{n \geq 1} H_{n} u<\frac{1}{2}
$$

on $X \backslash \bar{B}^{\prime \prime}$, where $B_{n}^{\prime \prime}$ is the relatively compact subregion of $X$ bounded by $\partial B_{n}$ so that it is obtained by attaching the chain of handles $S_{n}$ to $B_{n} \backslash \gamma_{n}$ crosswise along the slits $\gamma_{n}=\gamma_{n}^{\prime}$ and $B^{\prime \prime}:=\bigcup_{j \geq 1} B_{j}^{\prime \prime}$. Let

$$
T u=u-H u
$$

on $X \backslash \bar{S}$. It is clear that $T u \in H P\left(X \backslash \bar{S} ; \partial X \cup \gamma^{ \pm}\right)$and we instantly see that $T$ is a positively homogeneous additive mapping of $H P(X ; \partial X)$ onto $H P\left(X \backslash \bar{S} ; \partial X \cup \gamma^{ \pm}\right)$.

We first ascertain that $T: H P(X ; \partial X) \rightarrow H P\left(X \backslash \bar{S} ; \partial X \cup \gamma^{ \pm}\right)$is injective. Take two functions $u$ and $v$ arbitrarily in $H P(X ; \partial X)$ and assume that $T u=T v$, i.e. $u-H u=v-H v$. We have to show that $u=v$ on $X$. Since $H(u-v)=$ $u-v$ (cf. the remark for the extention of $H$ in the proof of (13)) is harmonic on each $B_{n}^{\prime \prime}$ and

$$
|H(u-v)| \leq H u+H v \leq \frac{u(a)}{2}+\frac{v(a)}{2}
$$

on $X \backslash B^{\prime \prime}$, we see that $|H(u-v)| \leq u(a) / 2+v(a) / 2$ on $X$. Since $H(u-v)$ has vanishing boundary values on $\partial X$, the maximum principle yields that $H(u-v)=$ 0 on $X$ so that $u-v=H(u-v)=0$ on $X$ and thus $u=v$ on $X$ as desired. 
To finish the proof of the relation (14) we need to do one more task: we show that the mapping $T: H P(X ; \partial X) \rightarrow H P\left(X \backslash \bar{S} ; \partial X \cup \gamma^{ \pm}\right)$is surjective. Take an arbitrary $v \in H P\left(X \backslash \bar{S} ; \partial X \cup \gamma^{ \pm}\right)$and we are to find a $u \in H P(X ; \partial X)$ such that $T u=v$. We can view that $v$ is subharmonic on $X$ by setting $v=0$ on $\bar{S}$. We may assume $v(a)=1$ this time too. Consider the function $w \in H P(X \backslash \bar{S}) \cap C(\bar{X})$ defined by

$$
w:=\sum_{n \geq 1} 4 k_{n} \omega\left(\cdot, \gamma_{n}^{ \pm}, X \backslash \bar{S}\right),
$$

where we have set that $\omega\left(\cdot, \gamma_{n}^{ \pm}, X \backslash \bar{S}\right)=1$ on $\bar{S}_{n}$ and $\omega\left(\cdot, \gamma_{n}^{ \pm}, X \backslash \bar{S}\right)=0$ on $\bar{S} \backslash \bar{S}_{n}$. Noting $\omega\left(\cdot, \gamma_{n}^{ \pm}, X \backslash \bar{S}\right)=\omega\left(\cdot, \gamma_{n}, \Omega \backslash \gamma\right)$ on $X \backslash \bar{S}=\Omega \backslash \gamma$, we see that $w \mid \gamma_{n}^{ \pm}=4 k_{n}$ and, by (7) and the definition of $k_{n}$, we deduce that

$$
w \mid \partial B_{n} \leq \sum_{v \geq 1} 4 k_{v} \cdot \frac{k_{v}}{2^{v+1} k_{v}^{2}}=2 .
$$

By considering partial sums we deduce that

$$
w \leq 2
$$

on $X \backslash \bar{B}^{\prime \prime}$. Therefore $s:=v+w \in H P(X \backslash \bar{S}) \cap C(\bar{X})$ and $s \mid \gamma_{n}^{ \pm}=4 k_{n}$, and

$$
s\left|\partial B_{n} \leq k_{n}+2 \leq 3 k_{n}<4 k_{n}=s\right| \gamma_{n}^{ \pm}
$$

implies that $s\left|B_{n}^{\prime \prime} \leq 4 k_{n}=s\right| \gamma_{n}^{ \pm}$, which shows that $s$ is a superharmonic majorant of $v$ on $X$. Hence there exists the least harmonic majorant $u$ of $v$ on $X$ such that $v \leq u \leq s$ on $X$. Hence in particular $u \in H P(X ; \partial X)$. Then, since $H u \leq H s=$ $H w=w$, we have

$$
|T u-v| \leq(u-v)+H u \leq(s-v)+H u \leq w+w=2 w
$$

on $X$. Observe that $T u-v=0$ on $\partial X \cup \gamma^{ \pm},|T u-v| \leq 2 w \leq 4$ on $X \backslash \bar{B}^{\prime \prime}$ so that $T u-v$ is bounded and harmonic on $X \backslash \bar{S}$ with vanishing boundary values, i.e. $T u-v=0$ on $\partial X \cup \gamma^{ \pm}$. Hence $T u=v$ and the proof of (14) is herewith complete.

Pick an arbitrary $u \in H P(\Omega \backslash \gamma ; \partial \Omega \cup \gamma)$ so that $u$ is nonnegative and harmonic on $\Omega \backslash \gamma$ and vanishing continuously on the relative boundary $\partial(\Omega \backslash \gamma)=\partial \Omega \cup \gamma$ of $\Omega \backslash \gamma$. Observe that $\Omega \backslash \gamma=X \backslash \bar{S}$ and the relative boundary $\partial(X \backslash \bar{S})$ of $X \backslash \bar{S}$ is $\partial X \cup \gamma^{ \pm}$. Therefore $u$ may be understood to be nonnegative and harmonic on $X \backslash \bar{S}$ and vanishing continuously on the relative boundary $\partial(X \backslash \bar{S})=\partial X \cup \gamma^{ \pm}$of $X \backslash \bar{S}$ so that $u \in H P\left(X \backslash \bar{S} ; \partial X \cup \gamma^{ \pm}\right)$. This natural identification $u \mapsto u$ gives a positively homogeneous and additive bijection of $H P(\Omega \backslash \gamma ; \partial \Omega \cup \gamma)$ to $H P(X \backslash \bar{S}$; $\left.\partial X \cup \gamma^{ \pm}\right)$. Hence we see that

$$
H P(\Omega \backslash \gamma ; \partial \Omega \cup \gamma) \simeq H P\left(X \backslash \bar{S} ; \partial X \cup \gamma^{ \pm}\right) .
$$

This with (13) and (14) assures that

$$
H P(\Omega ; \partial \Omega) \simeq H P(X ; \partial X) .
$$


Recall that $\Omega=R \backslash \bar{R}_{0} \quad$ with $\quad \partial \Omega=\partial R_{0} \quad$ and $\quad X=W_{R} \backslash \bar{R}_{0} \quad$ with $\quad \partial X=\partial R_{0}$. Therefore we finally conclude that

$$
H P\left(R \backslash \bar{R}_{0} ; \partial R_{0}\right) \simeq H P\left(W_{R} \backslash \bar{R}_{0} ; \partial R_{0}\right) .
$$

Let $\mathscr{M}(R)\left(\mathscr{M}\left(W_{R}\right)\right.$, resp. $)$ be the convex set $\left\{u \in H P\left(R \backslash \bar{R}_{0} ; \partial R_{0}\right): u(a)=1\right\}$ $\left(\left\{u \in H P\left(W_{R} \backslash \bar{R}_{0} ; \partial R_{0}\right): u(a)=1\right\}\right.$, resp.). We denote by $\mathscr{M}_{1}(R)\left(\mathscr{M}_{1}\left(W_{R}\right)\right.$, resp. $)$ the set of extreme points in the convex set $\mathscr{M}(R)\left(\mathscr{M}\left(W_{R}\right)\right.$, resp.). As another characterization of $\operatorname{dim} R$ (dim $W_{R}$, resp.) we recall that

$$
\operatorname{dim} R=\operatorname{card} \mathscr{M}_{1}(R) \quad\left(\operatorname{dim} W_{R}=\operatorname{card} \mathscr{M}_{1}\left(W_{R}\right), \text { resp. }\right) .
$$

Choose a positively homogeneous additive bijection $T$ giving (15) and consider the mapping

$$
u \mapsto \tau u:=\frac{1}{T u(a)} T u,
$$

where we see that $u>0$ is equivalent to $T u>0$ and a fortiori $\tau$ maps $\mathscr{M}(R)$ to $\mathscr{M}\left(W_{R}\right)$. We now prove that $\tau: \mathscr{M}(R) \rightarrow \mathscr{M}\left(W_{R}\right)$ is a bijection. First let $u$ and $v$ be arbitrarily chosen members in $\mathscr{M}(R)$ with $\tau u=\tau v$. Applying $T^{-1}$ to the both sides of $\tau u=\tau v$ we obtain $u / T u(a)=v / T v(a)$. Evaluating both sides of this at $a$ we see that $u(a) / T u(a)=v(a) / T v(a)$ and, since $u(a)=v(a)=1$, we have $1 / T u(a)=1 / T v(a)$ so that $u=v$. This proves that $\tau: \mathscr{M}(R) \rightarrow \mathscr{M}\left(W_{R}\right)$ is injective. Next take an arbitrary $v \in \mathscr{M}\left(W_{R}\right)$ and let $w:=T^{-1} v \in H P\left(R \backslash \bar{R}_{0} ; \partial R_{0}\right)$. Then $T w=v>0$ implies that $w>0$ and we can consider $u:=w / w(a) \in \mathscr{M}(R)$. Then, by $v(a)=1$, we see that

$$
\tau u=\frac{1}{T u(a)} T u=\frac{w(a)}{T w(a)} \cdot \frac{1}{w(a)} T w=\frac{1}{\left(T\left(T^{-1} v\right)\right)(a)} T\left(T^{-1} v\right)=\frac{1}{v(a)} v=v,
$$

i.e. $\tau u=v$ so that $\tau: \mathscr{M}(R) \rightarrow \mathscr{M}\left(W_{R}\right)$ is surjective. We have thus completed the proof of $\tau: \mathscr{M}(R) \rightarrow \mathscr{M}\left(W_{R}\right)$ being bijective.

Observe that the inverse mapping $T^{-1}$ of the bijection $T: H P\left(R \backslash \bar{R}_{0}: \partial R_{0}\right) \rightarrow$ $H P\left(W_{R} \backslash \bar{R}_{0} ; \partial R_{0}\right)$ in (15) is also additive and positively homogeneous along with $T$. Now take an arbitrary $v \in \mathscr{M}\left(W_{R}\right)$ and let $\tau^{-1} v=: u \in \mathscr{M}(R)$ or $v=$ $\tau u=(1 / T u(a)) T u$. Then $T^{-1} v=(1 / T u(a)) u$. Considering this identity at $a$ we see that $T^{-1} v(a)=1 / T u(a)$. Hence $\tau^{-1} v=u=T u(a) T^{-1} v$ and we obtain the following expression of $\tau^{-1}$ :

$$
v \mapsto \tau^{-1} v=\frac{1}{T^{-1} v(a)} T^{-1} v .
$$

Finally we prove that $\tau\left(\mathscr{M}_{1}(R)\right)=\mathscr{M}_{1}\left(W_{R}\right)$, which shows that $\tau: \mathscr{M}_{1}(R) \rightarrow$ $\mathscr{M}_{1}\left(W_{R}\right)$ is a bijection. Take any $u \in \mathscr{M}_{1}(R)$. We need first to show that $\tau u \in$ $\mathscr{M}\left(W_{R}\right)$ in fact belongs to $\mathscr{M}_{1}\left(W_{R}\right)$. Let $\tau u=\lambda v_{1}+(1-\lambda) v_{2}$ with $\lambda \in(0,1)$ and $v_{1}$ and $v_{2}$ being in $\mathscr{M}\left(W_{R}\right)$. Apply $T^{-1}$ to the both sides of the above identity and then we obtain $u=\mu u_{1}+\mu^{\prime} u_{2}$ with $\mu:=T u(a) \lambda T^{-1} v_{1}(a)$ and $\mu^{\prime}:=$ $T u(a)(1-\lambda) T^{-1} v_{2}(a)$ being positive numbers and $u_{1}:=\tau^{-1} v_{1}$ and $u_{2}:=\tau^{-1} v_{2}$ 
being in $\mathscr{M}(R)$. Considering the above identity $u=\mu u_{1}+\mu^{\prime} u_{2}$ at $a$ we obtain $1=\mu+\mu^{\prime}$ so that $u=\mu u_{1}+(1-\mu) u_{2}$ with $\mu \in(0,1)$. Since $u$ is extremal in $\mathscr{M}(R)$ (1.e. $\left.u \in \mathscr{M}_{1}(R)\right)$, we see that $u_{1}=u_{2}$, i.e. $\tau u=\lambda v_{1}+(1-\lambda) v_{2}$ implies $v_{1}=v_{2}$ and a fortiori $\tau u \in \mathscr{M}_{1}\left(W_{R}\right)$ or $\tau\left(\mathscr{M}_{1}(R)\right) \subset \mathscr{M}_{1}\left(W_{R}\right)$. Conversely choose any $v \in \mathscr{M}_{1}\left(W_{R}\right)$ and we are to show that $\tau^{-1} v \in \mathscr{M}(R)$ actually belongs to $\mathscr{M}_{1}(R)$. Let $\tau^{-1} v=\lambda u_{1}+(1-\lambda) u_{2}$ with $\lambda \in(0,1)$ and $u_{1}$ and $u_{2}$ in $\mathscr{M}(R)$. Apply $T$ to the both sides of the above by using $\tau^{-1} v=\left(1 / T^{-1} v(a)\right) T^{-1} v$ to deduce $v=\mu v_{1}+\mu^{\prime} v_{2}$ with $\mu:=T^{-1} v(a) \lambda T u_{1}(a)$ and $\mu^{\prime}:=T^{-1} v(a)(1-\lambda) T u_{2}(a)$ being positive numbers and $v_{1}:=\tau u_{1}$ and $v_{2}:=\tau u_{2}$ being in $\mathscr{M}\left(W_{R}\right)$. Considerring $v=\mu v_{1}+\mu^{\prime} v_{2}$ at $a$ we see that $1=\mu+\mu^{\prime}$ so that $v=\mu v_{1}+(1-\mu) v_{2}$ with $\mu \in(0,1)$. Since $v$ is extreme in $\mathscr{M}\left(W_{R}\right)$ (i.e. $v \in \mathscr{M}_{1}\left(W_{R}\right)$ ), we see that $v_{1}=v_{2}$ or $\tau u_{1}=\tau u_{2}$ and a fortiori $u_{1}=u_{2}$, which proves that $\tau^{-1} v \in \mathscr{M}_{1}(R)$, i.e. $\tau^{-1}\left(\mathscr{M}_{1}\left(W_{R}\right)\right) \subset \mathscr{M}_{1}(R)$ so that $\tau\left(\mathscr{M}_{1}(R)\right) \supset \mathscr{M}_{1}\left(W_{R}\right)$. Thus $\tau\left(\mathscr{M}_{1}(R)\right)=$ $\mathscr{M}_{1}\left(W_{R}\right)$ and $\tau: \mathscr{M}_{1}(R) \rightarrow \mathscr{M}_{1}\left(W_{R}\right)$ is bijective since $\tau: \mathscr{M}(R) \rightarrow \mathscr{M}\left(W_{R}\right)$ is bijective. Thus, by (15), we can conclude that

$$
\operatorname{dim} R=\operatorname{card} \mathscr{M}_{1}(R)=\operatorname{card} \tau\left(\mathscr{M}_{1}(R)\right)=\operatorname{card} \mathscr{M}_{1}\left(W_{R}\right)=\operatorname{dim} W_{R}
$$

so that the essential part of the proof of the main theorem is over. The proof of the main theorem as a whole is herewith complete.

\section{Planar parabolic surfaces}

A Riemann surface $R$ is said to be planar if it is represented as a subregion of the complex sphere $\hat{\mathbf{C}}: R \subset \hat{\mathbf{C}}$. Without loss of generality we can always suppose that the point at infinity $\infty$ of $\hat{\mathbf{C}}$ is contained in $R: \infty \in R$, which we always assume in the sequel. We also restrict ourselves to consider only planar surfaces $R$ which are open, i.e. noncompact so that the complement

$$
K:=\hat{\mathbf{C}} \backslash R
$$

is nonempty compact subset of $\hat{\mathbf{C}}$ whose complement $R$ is connected. For convenience we denote by $\mathscr{K}$ the class of nonempty compact subsets $K$ of the complex plane $\mathbf{C}:=\hat{\mathbf{C}} \backslash\{\infty\}$ with connected complement $\mathbf{C} \backslash K$ or equivalently $\hat{\mathbf{C}} \backslash K$ with respect to $\mathbf{C}$ or equivalently to $\hat{\mathbf{C}}$. Therefore the totality $\mathscr{P}$ of open planar Riemann surfaces $R$ and the totality $\mathscr{K}$ of nonempty compact subsets $K$ of $\mathbf{C}$ with connected complements are in bijective correspondence $R \mapsto K$ by the relation (17).

A compact subset $E \subset \mathbf{C}$ is said to be of (logarithmic) capacity zero if the energy integral over $E$ is infinite for any unit Borel measure $\mu$ on $E$ :

$$
\inf \iint \log \frac{1}{|z-w|} d \mu(z) d \mu(w)=+\infty,
$$

where the infimum is taken with respect to the family of Borel measures $\mu$ on $E$ with $\mu(E)=1$. It is off hand seen that $E \in \mathscr{K}$ if the capacity of $E$ is zero. We denote by $\mathscr{K}_{0}$ the class of all compact subsets $K$ of $\mathbf{C}$ which are of capacity zero. Then 


$$
\mathscr{K}_{0} \subset \mathscr{K} .
$$

A mapping $h: \hat{\mathbf{C}} \rightarrow[-\infty,+\infty]$ is said to be an Evans-Selberg potential for a given compact subset $E$ of $\mathbf{C}$ if the following three conditions are satisfied: $h \in H(\hat{\mathbf{C}} \backslash(E \cup\{\infty\})) ; h+\log \in H(\hat{\mathbf{C}} \backslash(E \cup\{0\}))$, where $\log$ is the function $z \mapsto$ $\log |z|$ with $\log \infty=\infty$;

$$
\lim _{z \in \hat{\mathbf{C}} \backslash E, z \rightarrow \zeta} h(z)=+\infty
$$

for every $\zeta \in E$. Hence in particular $h(\infty)=\lim _{z \rightarrow \infty} h(z)=-\infty$. Of course such an $h$ need not always exist for every given $E$, and in fact we see that $K \in \mathscr{K}_{0}$ if and only if there exists an Evans-Selberg potential $h$ for $K$. In terms of this characterization of the class $\mathscr{K}_{0}$ and the characterization of the class $O_{G}$ of parabolic surfaces by the existence of Evans function given in 33 , we obtain another important characterization of the class $\mathscr{K}_{0}$ : a planar open Riemann surface $R$ is parabolic if and only if $K$ in (17) belongs to $\mathscr{K}_{0}$, i.e.

$$
\mathscr{K}_{0}=\left\{K \in \mathscr{K}: \hat{\mathbf{C}} \backslash K \in O_{G}\right\} .
$$

This relation with the characterization (9) of the range set $\nabla$ in particular implies the following inclusion relation:

$$
\nabla \supset\left\{\operatorname{dim}(\hat{\mathbf{C}} \backslash K): K \in \mathscr{K}_{0}\right\} .
$$

Hence to complete the proof of Theorem A by the unified fashion we only have to show that the set on the right hand side of (19) above is identical with the set $\mathbf{N} \cup\left\{\aleph_{0}, \aleph\right\}$, which we will achieve in the sequel.

We fix an arbitrary $K \in \mathscr{K}_{0}$ and we are to study the Martin boundary $\Delta(R)$ and the Martin minimal boundary $\Delta_{1}(R)$ of $R:=\hat{\mathbf{C}} \backslash K$. We will show that the Martin compactification $R \cup \Delta(R)$ of $R$ is (topologically) identical with $\hat{\mathbf{C}}, \Delta(R)$ with $K$, and $\Delta_{1}(R)$ also with $K$. To see these we may assume without loss of generality that the disc $U:=\{z \in \mathbf{C}:|z|<1 / 2\}$ contains $K$, and we set $D:=$ $\{z \in \mathbf{C}:|z|<1\}$, the unit disc, so that $K \subset U \subset \bar{U} \subset D$. Let $g(z, w)$ be the Green function (kernel) on $V:=D \backslash K$. Since $K$ is of capacity zero, $g(z, w)$ can be uniquely extended to the Green function (kernel) on $D$ so that the Martin kernel on $R=D \backslash K$ is the restriction to it of the Martin kernel

$$
k(z, w):=\frac{g(z, w)}{g(c, w)}
$$

on $D$, where $c$ is an arbitrarily chosen point in $D \backslash K$. By this observation one instantly sees that the totality of Martin boundary points of $D \backslash K$ lying over $K$ is nothing but the set $K$ itself in $D$ so that we conclude that

$$
\Delta(R)=K, \quad R \cup \Delta(R)=\hat{\mathbf{C}} \quad(R=\hat{\mathbf{C}} \backslash K)
$$

(cf. (2) and (3) in $\S 1$ ). Hence a point $\zeta \in \Delta(R)=K$ belongs to the minimal Martin boundary $\Delta_{1}(R)$ if and only if $k(\cdot, \zeta)=\alpha g(\cdot, \zeta)(\alpha=1 / g(c, \zeta)$, a constant) is minimal, i.e. extreme in the convex set $\{u \in H P(D \backslash K ; \partial D): u(c)=1\}$. 
However, $g(\cdot, \zeta)$ is always minimal in $H P(D \backslash K ; \partial D)$ for every $\zeta \in K$ as a consequence of the Picard principle (cf. (5) in $§ 1$ ). Hence we have seen that

$$
\Delta_{1}(R)=K \quad(R=\hat{\mathbf{C}} \backslash K) .
$$

Since $\operatorname{dim} R=$ card $\Delta_{1}(R)=\operatorname{card} K$, we see that $\operatorname{dim}(\hat{\mathbf{C}} \backslash K)=\operatorname{card} K$ so that by (19) we conclude that

$$
\nabla \supset\left\{\operatorname{card} K: K \in \mathscr{K}_{0}\right\} .
$$

Finally we show that

$$
\left\{\operatorname{card} K: K \in \mathscr{K}_{0}\right\}=\mathbf{N} \cup\left\{\aleph_{0}, \aleph\right\},
$$

which with (22) implies (6) so that a unified proof for Theorem $A$ is here completed as announced. For each $n \in \mathbf{N}$, the finite set $K_{n}:=\{1,2, \ldots, n\}$ on the real line belongs to $\mathscr{K}_{0}$ and card $K_{n}=n$. Hence the set on the left hand side of (23) contains $\mathbf{N}$. Let $K_{\aleph_{0}}:=\{1 / n: n \in \mathbf{N}\} \cup\{0\}$. Clearly $K_{\aleph_{0}} \in \mathscr{K}_{0}$ and card $K_{\aleph_{0}}=\aleph_{0}$. We can pick a generalized 1-dimensional Cantor set $K_{\aleph}$ in $\mathscr{K}_{0}$ (cf. e.g. p. 336 in [11]). Since card $K_{\aleph}=\aleph$, we can now maintain $\{\operatorname{card} K: K \in$ $\left.\mathscr{K}_{0}\right\} \supset \mathbf{N} \cup\left\{\aleph_{0}, \aleph\right\}$. Conversely, choose an arbitrary compact set $K \subset \mathbf{C}$. The Cantor-Bendixson theorem says that $K$ is a union of a perfect set and a countable set, each of which may be empty. Since the cardinal number of a nonempty perfect set is $\aleph$, we see that card $K \in \mathbf{N} \cup\left\{\aleph_{0}, \aleph\right\}$ and in particular card $K$ : $\left.K \in \mathscr{K}_{0}\right\} \subset \mathbf{N} \cup\left\{\aleph_{0}, \aleph\right\}$. Hence we have established (23).

\section{Continuum hypothesis}

As the possible answers to the Heins problem on harmonic dimensions to determine the range set $\nabla=\{\operatorname{dim} R: R \in \mathscr{H}\}$, the following three typical cases are considered:

$$
\begin{gathered}
\nabla=[1, \aleph] ; \\
\nabla=\mathbf{N} \cup\left\{\aleph_{0}, \aleph\right\} ; \\
\mathbf{N} \cup\left\{\aleph_{0}, \aleph\right\}<\nabla<[1, \aleph],
\end{gathered}
$$

where $<$ indicates the strict inclusion relation, i.e. $A<B$ means that $A \subset B$ and $A \neq B$. If the continuum hypothesis is postulated, i.e. $\mathbf{N} \cup\left\{\aleph_{0}, \aleph\right\}=[1, \aleph]$, then Theorem A or (6) implies (24) or equivalently (25) and the Heins problem is completely settled. If the hypothesis is negated, then the above three cases are equally possible to hold at the present knowledge and the Heins problem should be said to be still widely open.

Let $\mathscr{X}$ be a class of open Riemann surfaces $R$ like $\mathscr{H}$ or $O_{G}$ and let us denote by $\nabla(\mathscr{X})$ the range set

$$
\nabla(\mathscr{X})=\{\operatorname{dim} R: R \in \mathscr{X}\}
$$

so that our original range set $\nabla$ is $\nabla=\nabla(\mathscr{H})=\nabla\left(O_{G}\right)$. It may be also interesting to determine $\nabla(\mathscr{X})$ for a given class $\mathscr{X}$ although there may not be any 
firm motivation to ask such a question like in the case of $\nabla=\nabla(\mathscr{H})=\nabla\left(O_{G}\right)$. Particularly important in this context is to determine $\nabla(\mathscr{X})$ for $\mathscr{X} \subset \mathscr{H}$ or $O_{G}$, which might open the way to the complete resolution of the Heins problem. Recall that $\mathscr{P}$ is the class of planar open Riemann surfaces. What we really did in $\S 5$ is that

$$
\nabla\left(O_{G} \cap \mathscr{P}\right)=\mathbf{N} \cup\left\{\aleph_{0}, \aleph\right\} .
$$

Let $\mathscr{C}$ be the class of multisheeted plane $R$, i.e. $\mathscr{C}$ is the family of every $R=$ $(R, \mathbf{C}, \pi)$, possibly branched covering surface of $\mathbf{C}$ with the projection $\pi$ such that card $\pi^{-1}(w)$ is a constant (sheet number) for every $w \in \mathbf{C}$. We also denote by $\mathscr{C}_{0}$ the subclass of finitely sheeted planes, i.e. $\mathscr{C}_{0}$ consists of $R \in \mathscr{C}$ with its sheet number in $\mathbf{N}$. What Heins did in [4] is

$$
\nabla\left(\mathscr{H} \cap \mathscr{C}_{0}\right)=\mathbf{N} .
$$

In this context the task to determine $\nabla(\mathscr{H} \cap \mathscr{C})$ is important. We know that $\nabla(\mathscr{H} \cap \mathscr{C}) \supset \mathbf{N} \cup\left\{\aleph_{0}, \aleph\right\}$ (unpublished) and essential question left here is to determine whether $\nabla(\mathscr{H} \cap \mathscr{C})=\mathbf{N} \cup\left\{\aleph_{0}, \aleph\right\}$ or not.

\section{REFERENCES}

[1] S. Axler, P. Bourdon and W. Ramey, Harmonic Function Theory, 2nd ed., Springer, 2001.

[ 2 ] C. Constantinescu and A. Connea, Über einige Problem von M. Heins, Rev. Math. pures Appl. 3 (1959), 277-281.

[3] A. CoRneA, Über eine Formel in der Extremisierungstheorie, Rev. Math. pures Appl. 3 (1958), 431-436.

[4] M. Heins, Riemann surfaces of infinite genus, Ann. of Math. 55 (1952), 296-317.

[5] Z. KuRAmochi, An example of a null-boundary Riemann surface, Osaka J. Math. 6 (1954), 83-91.

[6] M. NakaI, On Evans potential, Proc. Japan Acad., Ser. A. Math. Sci. 38 (1962), 624-629.

[7] M. Nakai and L. Sario, Harmonic and relative harmonic dimensions, Ann. Acad. Sci. Fenn., Ser. A. I. Math. 10 (1985), 419-432.

[8] I. RichaRds, On the classification of noncompact surfaces, Trans. Amer. Math. Soc. 106 (1963), 259-269.

[9] B. Rodin AND L. SARIo, Principal Functions, Van Nostrand, 1968.

[10] L. SARio AND K. Noshiro, Value Distribution Theory, Van Nostrand, 1966.

[11] L. SARIO AND M. NAKaI, Classification Theory of Riemann Surfaces, Springer, 1970.

[12] S. Segawa, A duality relation for harmonic dimensions and its applications, Kodai Math. J. 4 (1981), 508-514.

Mitsuru NAKAI

Professor Emeritus at:

Department of Mathematics

Nagoya Institute of TeChNOLOGY

Gokiso, SHOWA, NaGOYa 466-8555

JAPAN

E-mail address: nakai@daido-it.ac.jp 
Mailing address:

52 Eguchi, Hinaga

CHITA 478-0041

JAPAN

TOSHIMASA TADA

Department of Mathematics

School of Liberal Arts and Sciences

Daido Institute of Technology

TAKiharu, Minami, NagoYa 457-8530

JAPAN

E-mail address: tada@daido-it.ac.jp 